\title{
1 Molecular Mechanisms Governing Shade Responses in Maize
}

2 Qingbiao Shi ${ }^{\mathrm{a}, 1}$, Fanying Kong ${ }^{\mathrm{a}, 1}$, Haisen Zhang ${ }^{\mathrm{a}, 1}$, Yu'e Jiang ${ }^{\mathrm{a}}$, Siqi Heng ${ }^{\mathrm{a}}$, Ran Liang ${ }^{\mathrm{a}}$,

3 Jisheng $\mathrm{Liu}^{\mathrm{b}}$, Xiaoduo Lu ${ }^{\mathrm{b}}$, Pinghua $\mathrm{Li}^{\mathrm{c}}$, Gang Li $\mathrm{Li}^{\mathrm{a}, 2}$

4

$5 \quad{ }^{a}$ College of Life Science, State Key Laboratory of Crop Biology, Shandong Agricultural

6 University, Tai'an, Shandong 271018, China

$7 \quad{ }^{b}$ College of Life Science, Qilu Normal University, Jinan, 250013, China

$8 \quad{ }^{c}$ College of Agronomy, State Key Laboratory of Crop Biology, Shandong Agricultural

$9 \quad$ University, Tai'an, Shandong 271018, China

10

$11{ }^{1}$ Q.S., F.K., and H.Z. contributed equally to this work.

$12 \quad{ }^{2}$ To whom correspondence may be addressed. Email: gangli@sdau.edu.cn

The authors declare no conflict of interest.

Running title: Shade responses in maize

Highlight: Our findings not only increase the understanding of the regulatory network

19 of the shade avoidance in maize, and also provide a useful resource for maize genetics and breeding. 


\section{Abstract}

Light is one of the most important environmental factors affecting plant growth and development. Plants use shade avoidance and shade tolerance strategies to adjust their growth and development thus increase their success in the competition for incoming light. To investigate the mechanism of shade responses in maize (Zea mays), we examined the anatomical and transcriptional dynamics of the early shade response in seedlings of the B73 inbred line. Transcriptome analysis identified 912 differentially expressed genes, including genes involved in light signaling, auxin responses, and cell elongation pathways. Grouping transcription factor family genes and performing enrichment analysis identified multiple types of transcription factors that are differentially regulated by shade and predicted putative core genes responsible for regulating shade avoidance syndrome. For functional tests, we ectopically overexpressed $Z m H B 53$, a type II HD-ZIP transcription factor gene significantly induced by shade, in Arabidopsis thaliana. Transgenic Arabidopsis plants overexpressing ZmHB53 exhibited narrower leaves, earlier flowering, and enhanced expression of shade-responsive genes, suggesting that ZmHB53 participates in the regulation of shade responses in maize. This study increases our understanding of the regulatory network of the shade response in maize and provides a useful resource for maize genetics and breeding.

Key words: shade avoidance syndrome, RNA-seq, HD-ZIP transcription factor, maize 


\section{Introduction}

Light plays a fundamental role in plant growth and development. Increasing the planting density of crops, particularly grasses such as maize (Zea mays), to increase yield is a common practice in modern agriculture. However, under high-density cultivation, light, water, and nutrients limit plant growth and seed production. Blue and red wavelengths light are preferentially absorbed by photosynthetic pigments of the upper leaves of the canopy for photosynthesis, resulting in a reduction of photosynthetically active radiation (PAR), and low ratio of red to far-red (R:FR) light in the lower leaves. In most plant species, the reduction of PAR, low R:FR and low blue light act as shade signals to induce shade avoidance syndrome (SAS), including elongation of stems and petioles and inhibition of the outgrowth of axillary buds, thus allowing plants to reach light and shade their neighbors (Keuskamp et al., 2010; Sharwood et al., 2014; Ballaré et al., 2017; Pignon et al., 2017). Long-term shade treatments lead to severe SAS and significantly decrease seed production (Casal, 2013); for example, maize grain yield may be reduced by up to $60 \%$ by long-term shade treatment (Cui et al., 2015). Therefore, understanding shade avoidance responses and improving plant success in the competition for light, without decreasing yields, are important goals to improve high-density planting of crops (Maddonni et al., 2001; Page et al., 2010).

The molecular network regulating SAS has been well documented in Arabidopsis thaliana. Various shade signals are primarily perceived by photoreceptors, including phytochromes and cryptochromes. Under high R:FR light, phytochromes (mostly phyB in Arabidopsis) enter the nucleus in the active form (far red-absorbing form, $\mathrm{Pfr}$ ) and regulate numerous downstream genes, thereby suppressing the shade response (Kircher et al., 1999; Franklin, 2008; Chen et al., 2011). Low R:FR increases the ratio of inactive phyB (red-absorbing form, $\mathrm{Pr}$ ) in the cytosol, thus releasing the inhibition of downstream signaling components and promoting the shade response (Kircher et al., 1999). The Arabidopsis phyb mutant and the maize phyb1 phyb2 double mutant exhibit a constitutive SAS phenotype, including slender petioles, leaves, and accelerated stem elongation (Robson et al., 1993; Sheehan et al., 2007). Branch formation is also 
inhibited in the early development of phyb mutants in sorghum (Kebrom et al., 2016).

PHYTOCHROME INTERACTING FACTORS (PIFs) act as important downstream signal transduction components of phytochromes and play a key role in SAS (Castillon et al., 2007; Leivar et al., 2011). Arabidopsis plants overexpressing PIF4, PIF5, and PIF7 exhibit constitutive SAS under high R:FR conditions (Lorrain et al., 2008; Li et al., 2012). Consistent with this, the pifq (pif1 pif3 pif4 pif5) quadruple mutant and pif7 mutants show short petioles and a reduced response to shade (Leivar et al., 2008; Li et al., 2012). Overexpressing ZmPIF4 in Arabidopsis also produces constitutive SAS (Shi et al., 2018). Analyses of genome-wide downstream targets revealed that PIFs directly target hundreds of growth-promoting genes, such as Aux/IAA (IAA19, IAA29), YUCCA (YUC2, YUC5, YUC8, YUC9), EXPANSINS (EXPA1, EXPB1) and XYLOGLUCAN ENDOTRANSGLYCOSYLASE/HYDROLASE (XTH15, XTH33) (Zhang et al., 2013; Pfeiffer et al., 2014). In addition, the contents and sensitivities of free auxin, gibberellin (GA) and brassinosteroids were rapidly induced by shade treatments, thus promoting cell elongation in Arabidopsis (Bou-Torrent et al., 2014), bean (Beall et al., 1996) and sunflower (Kurepin et al., 2007).

In Arabidopsis, shade treatments rapidly induce the expression of many transcription factor genes, including LONG HYPOCOTYL IN FAR-RED (HFR1), PHYTOCHROME RAPIDLY REGULATED GENE 1 (PAR1), PAR2, and PIF3-LIKE1 (PIL1), which encode basic helix-loop-helix (bHLH) type transcription factors that negatively regulate PIF activities through physical interactions, thereby preventing an exaggerated shade response (Roigvillanova et al., 2006; Hornitschek et al., 2009; Hornitschek et al., 2012). Additionally, multiple homeodomain leucine zipper (HD-ZIP) and B-box (BBX) type transcription factors function in the shade response (Sorin et al., 2009; Gangappa et al., 2014).

In maize, although some of the early shade-responsive genes have been identified (Wang et al., 2016), their physiological functions and underlying mechanisms remain largely unknown. Here, we combined cytological and transcriptomic analysis with functional testing to investigate the anatomical and transcriptional dynamics of SAS in maize seedlings and predict the core responsive genes involved in the 
regulation of SAS.

\section{Materials and Methods}

\section{Plant materials and growth conditions}

Seedlings of maize inbred lines were grown in growth chambers under a 12-hour light/12-hour dark cycle at $180 \mu \mathrm{mol} / \mathrm{m}^{2} / \mathrm{s}$ of light intensity at $25{ }^{\circ} \mathrm{C}$. For short-term simulated shade treatment, seedlings of B73 (V3 stage) were transferred from white light to constant FR light $\left(10.52 \mu \mathrm{mol} / \mathrm{m}^{2} / \mathrm{s}\right)$ for 0,1 , and $3 \mathrm{~h}$, followed by constant $\mathrm{R}$ light $\left(50 \mu \mathrm{mol} / \mathrm{m}^{2} / \mathrm{s}\right)$ for $1 \mathrm{~h}$ and then used for qPCR and RNA-seq assays. For longterm simulated shade treatment (Figure 1 and S1), various inbred lines were grown under white light $\left(65.6 \mu \mathrm{mol} / \mathrm{m}^{2} / \mathrm{s}\right)$ supplied with FR light $\left(10.52 \mu \mathrm{mol} / \mathrm{m}^{2} / \mathrm{s}\right.$, low $\left.R: F R\right)$, or $\mathrm{R}$ light $\left(50.0 \mu \mathrm{mol} / \mathrm{m}^{2} / \mathrm{s}\right.$, high $\left.\mathrm{R}: \mathrm{FR}\right)$ after seed germination. After shade treatment, scanning electron microscopy (SEM) of sheath and leaf blade tissues were performed as previously described (Kong et al., 2017).

The Arabidopsis thaliana wild-type control plants used in this study were ecotype Columbia-0 (Col-0). The seeds were surface-sterilized with $20 \%$ bleach for 20 min and washed four times with sterile $\mathrm{dd} \mathrm{H}_{2} \mathrm{O}$. After being stratified for two days at $4{ }^{\circ} \mathrm{C}$, the seeds were germinated on germination medium (GM) plates.

\section{$R T-q P C R$}

Total RNA was extracted using an Ultrapure RNA kit (CWBIO, Beijing). The reversetranscription reactions were performed using an AMV reverse transcriptase (Fermentas). The RT-qPCR was performed on a 7500 Fast Real-Time PCR machine (ABI) using SYBR Real Master Mix (Tiangen, Beijing). Primers used for RT-qPCR are listed in Table S3.

\section{RNA-seq analysis}

The cDNA library construction, sequencing, and data analyses were performed as described previously (Kong et al., 2017). The maize B73 reference genome (AGPv3.22) were used for mapping the reads. The Cufflinks 2.2.1 package was used to calculate the gene expression levels with the parameter of reads per kilobase per million 
mapped reads (RPKM) and detect differentially expressed genes (DEGs) using default parameters. The false discovery rate (FDR) was used to determine the threshold of the $p$-value in multiple tests. A threshold of FDR $\leqslant 0.05$ and a fold change $\geqslant 2$ were used to judge the significance of differences in gene expression.

\section{Cluster and functional enrichment analysis}

DEGs that were commonly expressed under both FR light and after re-exposure to $R$ light (Dataset S2) and the expressed transcription factor genes were subjected to cluster analysis (Dataset S1). The RPKM values (normalized to the maximum of all RPKM values of the gene in B73 seedlings treated with FR light for $0 \mathrm{~h}, 1 \mathrm{~h}$ or $3 \mathrm{~h}$, followed by $\mathrm{R}$ light for $1 \mathrm{~h}$ ) were subjected to cluster analysis using the K-Means Support (KMS) module in the MultiExperiment Viewer (MEV) program.

\section{Plasmid construction and generation of transgenic Arabidopsis plants}

To generate transgenic ZmHB53-OE lines in the Arabidopsis Col-0 background, the coding region of $Z m H B 53$ was PCR-amplified from cDNA of inbred line B73 using the primers pair ZmHB53-F and ZmHB53-R (Table S3). Then, ZmHB53 fragment was inserted into the BamHI and Xbal digested pPZP211-35Spro::3FLAG binary vector (Ma et al., 2017) to produce 35Spro::ZmHB53-3FLAG. More than 20 independent transgenic lines were selected and verified by RT-qPCR, followed by immunoblot analysis as described previously (Ma et al., 2016).

\section{Results}

\section{Low R:FR induces the SAS in maize seedlings}

To investigate the effects of shade on maize growth, seedlings of various inbred lines were grown under white light supplied with FR ( $R$ :FR ratio 0.19 ) or $R$ ( $R: F R$ ratio13.3) conditions. After simulated shade treatment, the mesocotyl length, leaf length, and plant height significantly increased in inbred lines B73, Mo17, Huangzao4, Zheng58 and Su115, compared to plants under high R:FR conditions (Fig. 1a, 1b, S1). Mesocotyl length increased more strongly in inbred B73 (by 17\%) and Mo17 (20\%), compared with the other inbred lines (Fig.1b, S1). Moreover, the inbred lines 178 and 
Q319 were less responsive to simulated shade-induced elongation of mesocotyls and plant height, compared to the inbred lines B73 and Mo17 (Fig. 1). In addition, anthocyanin accumulation obviously decreased in the base region of the sheath in all the tested inbred lines under low R:FR conditions, compared with control plants grown under high R:FR conditions (Fig.1a, S1a).

To investigate the effects of simulated shade on cell elongation in B73, we observed the epidermal cells of the leaf blade and sheath by SEM. As shown in Figure 1c, cell elongation in the leaf blade increased slightly, while cell elongation in the sheath increased substantially after shade treatment. To further explore the effects of supplemental FR on cell elongation, we examined the transcript levels of cell elongation-related genes in V3 stage B73 and 178 seedlings that were transferred from white light to FR light for 1 and $3 \mathrm{~h}$, followed by $1 \mathrm{~h}$ in red light. The transcript levels of $X T H 8, X T H 23$, and EXPB2 were significantly induced by FR light and repressed by $\mathrm{R}$ light in inbred B73, but showed no obvious change in 178 (Fig. 1d), consistent with its reduced sensitivity to simulated shade treatment in Figure 1a-b.

Generation and analysis of RNA-seq data for treated plants

To gain insight into the molecular regulatory mechanism of the shade response in maize, we conducted global RNA-seq of B73 seedlings at the V3 stage treated with FR light for $0 \mathrm{~h}$ (F0), $1 \mathrm{~h}$ (F1), or $3 \mathrm{~h}$ (F3), followed by $\mathrm{R}$ light for $1 \mathrm{~h}$ (R1) (short-term shading treatment). Using paired-end Illumina sequencing, we generated sequences from eight libraries (four time points with two biological replicates), producing approximately 1.9 billion high-quality reads, $95 \%$ of which uniquely mapped to the B73 reference genome, version 3 . The distribution of reads was $75.8 \%$ in exons, $9.3 \%$ in introns, and $11.2 \%$ in intergenic genomic regions (Table S1). Comparisons of the biological replicates showed that their expression values were highly correlated (average $\mathrm{R}^{2}=0.963$, Fig. S2), indicating that the results of biological replicates in this study are highly reproducible. To reduce the influence of transcriptional noise, genes from the B73 filtered gene set (FGS) were included for analysis only if their RPKM values were $\geqslant 1$. In total, 22,479 genes were expressed under at least one condition, 
including 18,968 (84.5\%) genes commonly expressed among all four conditions (Fig. 2a, Dataset S1).

To verify the quality of RNA-seq data, we performed RT-qPCR analyses of 48 transcripts, revealing a high correlation $\left(R^{2}=0.587\right)$ between the RNA-seq and RTqPCR data (Fig. S3b). As expected, ZmphyA1, ZmphyB1, ZmphyB2, ZmphyC1, and ZmphyC2 were significantly induced by FR (Fig. S3a). ZmHY5 was strongly downregulated by FR. Additionally, multiple genes encoding proteins involved in the light reactions in photosynthesis, such as $Z m L H C B 1, Z m P S B A, Z m P S B Q$, and $Z m P S B 28$ were downregulated by FR (Fig. S3a).

Further, we identified 327, 591, and 195 DEGs between F0 and F1 (F1 vs. F0), F0 and F3 (F3 vs. F0) and F3 and R1 (R1 vs. F3), respectively (Fig. 2b and c, Dataset S2). Among these, 111 genes were common between F1 vs. F0, and F3 vs. F0, including three genes showing opposite expression patterns. Therefore, after excluding these three oppositely expressed genes, a total of 804 FR-regulated DEGs were identified (Fig. 2b). Interestingly, among the 87 common DEGs between FRregulated, and red-regulated (R1 vs. F3), 80 (92\%) genes showed opposite expression patterns, suggesting that most of the effects of FR light on gene expression can be reversed by subsequent treatment with $\mathrm{R}$ light (Fig. 2c). All these DEGs (912 genes) were selected as putative conserved genes important for the SAS in maize.

\section{Dynamics of gene expression during the SAS in maize}

To better understand the regulatory network of the SAS in maize, we further grouped these 912 genes into 10 clusters $(\mathrm{C} 1-\mathrm{C} 10)$ based on their expression patterns and then subjected to MapMan functional enrichment analysis (Fig. 2c). Among clusters (C1-C3) with reduced expression by FR, the most highly enriched categories included genes encoding proteins that mediate the light reactions, sucrose synthesis, and secondary metabolic pathways (Fig. 2e). For example, most of the anthocyanin biosynthesis related genes, including $\mathrm{CHS}, \mathrm{CHI}, \mathrm{F} 3 \mathrm{H}, \mathrm{DFR}$, and $\mathrm{ANS}$ were highly downregulated by FR (Fig. S4), consistent with the reduced anthocyanin accumulation in shade-treated plants (Fig. 1a). 
Among clusters of genes whose expression was induced by FR (C4-C10), the most highly enriched categories included genes encoding proteins involved in cell wall modification, degradation of starch and sucrose, hormone metabolism, and various signal pathways, suggesting they might play important roles in early responses to shade in maize. For example, genes related to auxin biosynthesis (e.g., ZmYUC5 and ZmTAA1), and ethylene signal transduction (e.g., ZmERF7) were significantly induced by FR and downregulated by subsequent R treatment (Fig. 2e, S5). Interestingly, most of the alterations in expression (up- or downregulation) induced by FR were reversed by subsequent $\mathrm{R}$ treatment in most clusters, except for $\mathrm{C} 10$, which was enriched for genes involved in vitamin metabolism, protein targeting, and signaling. All these results are consistent with the regulatory network controlling the SAS in Arabidopsis (Li et al., 2011), suggesting that the regulatory mechanism of the SAS is evolutionarily conserved between monocots and dicots.

Transcription factors play important roles in the maize SAS

Of the 3,316 maize transcription factor genes identified in the Plant Transcription

231 Factor Database (http://planttfdb.cbi.pku.edu.cn/), 1,353 (41\%) were commonly expressed under all four treatment conditions (Dataset S1). These genes were further classified into five groups based on their expression patterns (G1-G5, including 262, 212, 450, 191, and 238 genes, respectively; Fig. 3a). Shade-downregulated transcription factors were grouped into G1 and G2, including the HD-ZIP (21/43 expressed HD-ZIPs were included in G1 and G2) and MYB (44/124) transcription factors (Fig. 3b). Early shade-induced transcription factors were grouped into G3, which was significantly enriched for bHLH (41/109), ERF (46/92) and GRAS (21/48) family members (Fig. 3b, Dataset S1). Some bHLH family genes, including members of the PIF sub-family (ZmPIF3, ZmPIF5, and PIF-like) were rapidly induced by FR treatment. In addition, atypical PIF family genes, including ZmHFR1, ZmPAR1, ZmPAR2 and ZmPIL1, were rapidly induced by shade and might play a negative role in the SAS (Dataset S1). Late shade-induced transcription factors were grouped in G4 and G5, and were significantly enriched for ARF (15/24) and HB/other (9/15) family 
members.

\section{Core genes involved in regulating the SAS}

To identify the important regulators of the shade response, we first listed the 226 genes overlapping in our DEG list (912, Dataset S2) and Wang's DEG list (1105, Wang et al., 2016), eliminated the photosynthesis, secondary metabolism, stress, nucleotide metabolism, and function unknown genes from this list, added three genes, ZmGT1 (Grassy tillers1), ZmTB1 (Teosinte branched1), and ZmVT2, which have already been shown to play important roles in maize SAS (Doebley et al., 1997; Sheehan et al., 2007; Phillips et al., 20011; Whipple et al., 2011), and identified 93 core genes for the shade response (Table1, S2). Most of these genes were significantly regulated by shade treatment. In addition to ZmGT1, ZmTB1, and ZmVT2, ZmphyB1 and ZmphyB2 have also been proved to participate in maize SAS (Sheehan et al., 2007). The other core shade-responsive genes have not previously been shown to directly regulate the SAS in maize, but are related to light signaling, hormone metabolism and signal transduction, regulation of transcription, cell wall modification, protein metabolism and so on (Table 1). For example, multiple plant hormone-related genes including IAAs, SAURs and GH3.1, GA1, GA5, GA20x1, GA20x8, CKX6, ACO1, EIN4 and ERFs were identified, suggesting that they may play crucial roles in the SAS in maize (Table 1). Interestingly, we identified 7 BBXs as core genes for SAS regulation (Table 1, S2). For example, $Z m B B X 20$ was upregulated 2.9 -fold (F1 vs. F0) and 12.7-fold (F3 vs. F0) in response to shade treatment in the current study, and 2.5 -fold ( $1 \mathrm{~h}$ vs. $0 \mathrm{~h}$ ), 2.1 -fold ( 3 $\mathrm{h}$ vs. $0 \mathrm{~h}$ ) and 2.9-fold (6 h vs. $0 \mathrm{~h}$ ) in the previous study (Wang et al., 2016).

\section{$Z m H D$-ZIP proteins act as regulators of the SAS}

Research in Arabidopsis has shown that HD-ZIP transcription factors modulate the SAS (Sorin et al. 2009). In our RNA-seq data, many HD-ZIP family genes were up- or downregulated by shade, therefore, this transcription factor family was selected for further analysis (Fig. 4a). Phylogenetic analysis of this family genes in Arabidopsis and maize revealed that these genes were classified into the I, II, III and IV subfamilies (Fig. 4b). Interestingly, one-third of type I HD-ZIP and all the type II HD-ZIP genes were up 
regulated, while two-thirds type I HD-ZIP genes were down regulated by FR (Fig. 4a), indicating that various members of this transcription factor family (for example type I and II HD-ZIP genes) might play opposite roles in the shade response. Consistent with the results of RNA-seq, qPCR analyses revealed that type II HD-ZIPs, including ZmHB4, ZmHB53, ZmHB59, ZmHB78, and ZmHB86, were strongly induced by FR, and subsequently suppressed by R light; by contrast, type I HD-ZIPs, such as ZmHB34, $Z m H B 66$, and $Z m H B 70$, were slightly reduced by FR and induced by $\mathrm{R}$ light (Fig. 4c). These results demonstrate that both the type I and II HD-ZIP subfamily members might play more important roles in the responses to shade signals.

We further measured the expression levels of these HD-ZIP genes in the B73 and 178 inbred lines under simulated shade conditions. As shown in Figure 4d, ZmHB43, ZmHB53, ZmHB78 and ZmHB127 were induced by shade in both inbred B73 and 178 , while $Z m H B 34, Z m H B 66$, and $Z m H B 70$ showed opposite expression patterns in these inbred lines under shade treatment, suggesting these genes may contribute to the differential response to shade between the B73 and 178 inbred lines.

\section{ZmHB53 can affect the shade response in maize}

To further investigate the roles of HD-ZIPs in the shade response, we focused on ZmHB53 (GRMZM2G044752), a homolog of ATHB4 which is essential in shade response and leaf development in Arabidopsis (Sorin et al. 2009). To investigate whether ZmHB53 affects leaf morphogenesis and shade responses, we overexpressed a FLAG-tagged version of ZmHB53 (ZmHB53-3Flag) under the control of the constitutive $35 \mathrm{~S}$ promoter in the Arabidopsis Col-0 background. Three independents transgenic ZmHB53 overexpression lines (OE5, OE6, and OE8) were selected based RT-qPCR and western-blotting, and then subjected to further physiological analysis (Fig. S5a).

All three lines exhibited a slight SAS, including narrow rosette leaves and early flowering time, compared with wild-type Col-0 plants under long-day (LD, 16-hour light/8-hour dark) conditions (Fig. 5a-b, 5d-e). Interestingly, the transgenic lines had more branches and reduced plant height compared to wild type at the mature stage, 
representing a lessened response to shade treatment, compared with wild-type control plants (Fig. 5c, 5e), indicating that ZmHB53 can affect SAS in Arabidopsis via a complex regulatory mechanism. However, in seedlings grown under dark, white, and low R/FR light conditions, Arabidopsis $\mathrm{ZmHB53}$ overexpression lines showed no significant differences from wild-type control plants (Fig. S5), suggesting that $Z m H B 53$ mainly functions at the mature stage.

Next, we examined the transcript levels of genes that respond rapidly to shade

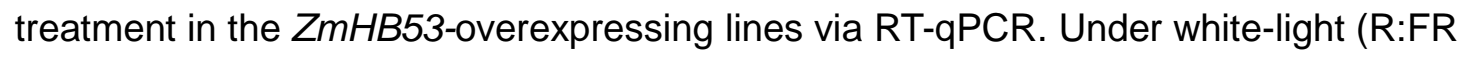
7.8) conditions, the transcript levels of well-known shade-responsive genes including HFR1, PAR1, PIL1, and EXP2, all significantly increased in the Arabidopsis ZmHB53overexpressing lines, compared with wild-type Col-0 control plants (Fig. 5f). After simulated shade treatment $(\mathrm{W}+\mathrm{FR})$, the expression of HFR1, PAR1, and PIL1, were significantly upregulated in Col-0 and $\mathrm{ZmHB53}$ overexpression lines, compared with control plants under white light conditions (Fig. $5 f-g$ ). All these results indicate that overexpressing $Z m H B 53$ enhances the transcript levels of shade-response genes in Arabidopsis.

\section{Discussion}

Light is one of the essential factors determining yield potential in the modern highdensity cultivation of crop plants. In most plant species showing shade avoidance response, changes in light quantity and quality cause morphological responses including elongated stems and petioles, and more erect leaf angle; these responses increase leaf vertical inclination and help the plant compete for light (Zhu et al., 2014; Bongers et al., 2018). Here, we found that the maize inbred lines 178 and Q319 exhibited less-pronounced responses to simulated shade treatment, compared with inbred lines B73 and Mo17 (Fig.1a-b). B73 maize seedlings under simulated shade conditions showed typical symptoms of the SAS, such as elongated mesocotyls, stems, and leaves, and reduced accumulation of anthocyanin (Fig. 1a-b). Consistent with this, cytological, qPCR and RNA-seq analyses showed that simulated shade treatment 
induced the transcription levels of cell elongation-related genes and promoted cell elongation in leaf blades and sheaths (Fig. 1c-d).

Phytochrome signaling pathways play a conserved role in the low R:FR induced shade response in both maize and Arabidopsis (Lee et al., 2017). Arabidopsis PIF4, PIF5 and PIF7 act as the downstream signal transduction components of multiple photoreceptors (including phytochromes and cryptochromes) and play crucial roles in shade responses (Lorrain et al., 2008; Leivar et al., 2011; Li et al., 2012). Here, ZmphyA1, ZmphyB1, ZmphyB2, and five PIF family genes were all upregulated by FR, suggesting that they may play important roles in shade responses. Consistent with this, our previously study showed that the over-expression of ZmPIF4 and ZmPIF5 causes a constitutive shade avoidance response in Arabidopsis, indicating that they might play essential roles in shade responses in maize (Shi et al. 2018).

A reduction in the outgrowth of axillary buds is one of the typical morphological changes of the shade avoidance response. The Arabidopsis TCP (TEOSINTE BRANCHED 1, CYCLOIDEA, PCF) type transcription factor BRANCHED 1 (BRC1) directly binds to and activates the transcription of a group of HD-ZIP I transcription factor genes, including $H B 21, H B 40$, and $H B 53$, thus preventing constitutive outgrowth of branches (Gonzalez-Grandio et al., 2017). Maize TB1 is a homolog of BRC1, and negatively regulates the outgrowth of axillary buds (Doebley et al., 1997). Maize GT1, encoding an HD-ZIP I family member, is one of the downstream targets of TB1 and represses the outgrowth of lateral buds (Whipple et al., 2011). Therefore, it appears that the genetic module involving the BRC1/TB1 and HD-ZIP transcription factors is evolutionarily conserved in dicots and monocots, where it prevents branching under light-limiting conditions. Interestingly, the Arabidopsis ZmHB53 (HD-ZIP II) overexpression lines showed more branches than the wild-type control plant, which contrasts with the phycological function of maize GT1 (Fig. 5). Therefore, we hypothesized that HD-ZIP transcription factors, for example HD-ZIP I and II sub-family, may play negative and positive roles in regulating the outgrowth of axillary buds, respectively, like the functions of bHLH type transcription factors in SAS, such as the positive roles of PIF4 and PIF5, and the negative roles of HFR1, PAR1, and PAR2 in 
362 the shade response in Arabidopsis. This is also consistent with the opposite expression

363 trends of type I and II HD-ZIP genes in response to shade in maize (Fig. 4a).

364 In summary, the monocotyledonous plant maize and the dicotyledonous plant 365 Arabidopsis share a number of morphological and physiological responses in their 366 shade responses. When plants are exposed to shade conditions, photoreceptor 367 systems perceive a reduction of PAR, low ratio of $R: F R$, as well as low blue light, and subsequently activate a downstream network of various interacting transcriptional regulators and hormones to adjust plant growth and development to increase the plant's ability to compete for light (Fig. S6). Based on this model, three different strategies could be developed to increase the ability of maize to compete for light and minimize the negative effects of the SAS. In the upper regulatory layer, one strategy could involve modulating the expression levels or activities of photoreceptor genes such as ZmphyA1, ZmphyA2, ZmphyB1, and ZmphyB2, as they directly respond to dynamic environmental light changes. At the middle regulatory layer, another strategy could modify the expression of important regulators of the SAS, such as ZmPIF4, ZmPIF5, ZmHFR1 and ZmHB53. In the downstream regulatory layer, a third strategy could modify the expression levels of many SAS-related genes, including those directly involved in cell elongation, hormone synthesis, or signaling transduction, such as ZmTAA1 and ZmYUC5. Finally, our study identified a core set of shade-responsive genes, which expands the regulatory network of shade responses and provides a useful resource for maize genetics and breeding. 


\section{Supplemental Information}

Supplemental information is available online.

Fig. S1. The phenotype of maize plants grown under high or low R:FR conditions.

Fig. S2. Correlation between biological replicates.

Fig. S3. Verification of RNA-seq results by RT-qPCR.

Fig. S4. Expression analysis of genes involved in anthocyanin biosynthesis in maize by RNA-seq.

Fig. S5. Identification of $Z m H B 53$ overexpression transgenic plants and the response of seedlings to shade.

Fig. S6. Model of the putative regulatory network of the early shade response in maize. Table S1. RNA-seq data analysis.

395 Table S2. Core responsible genes involved in regulating the shade response in maize.

396 Table S3. Primers used in this study.

397 Dataset S1. Genes expressed during the shade response in maize.

398 Dataset S2. Differentially expressed genes during the shade response in maize.

\section{Acknowledgments}

401 This work was supported by grants from the State Key Basic Research and 402 Development Program of China (2014CB147301 and 2016YFD0101003), the National 403 Key Research and Development Plan of China (2017YFD0301001 and 404 2018YFD0300603), the National Natural Science Foundation of China (31701434), 405 Funds of Shandong "Double Tops" Program (to GL) and the State Key Laboratory of 406 Crop Biology (DXKT201706). 


\section{References}

Ballaré CL, Pierik R. 2017. The shade-avoidance syndrome: multiple signals and ecological consequences. Plant, Cell and Environment 40, 2530-2543.

Beall FD, Yeung EC, Pharis RP. 1996. Far-red light stimulates internode elongation, cell division, cell elongation, and gibberellin levels in bean. Revue Canadienne De Botanique 74, 743-752.

Bongers FJ, Pierik R, Anten NPR, Jochem B, Evers JB. 2018. Subtle variation in shade avoidance responses may have profound consequences for plant competitiveness. Annals of Botany 121, 863-873.

Bou-Torrent J, Galstyan A, Gallemi M, Cifuentes-Esquivel N, Molina-Contreras MJ, SallaMartret M, Jikumaru Y, Yamaguchi S, Kamiya Y, Martinez-Garcia JF. 2014. Plant proximity perception dynamically modulates hormone levels and sensitivity in Arabidopsis. Journal of Experimental Botany 65, 2937-2947.

Casal JJ. 2013. Photoreceptor signaling networks in plant responses to shade. Annual Review of Plant Biology 64, 403-427.

Castillon A, Shen H, Huq E. 2007. Phytochrome interacting factors: central players in phytochromemediated light signaling networks. Trends in Plant Science 12, 514-521.

Chen M, Chory J. 2011. Phytochrome signaling mechanisms and the control of plant development. Trends in Cell Biology 21, 664-671.

Cui H, Camberato JJ, Jin L, Zhang J. 2015. Effects of shading on spike differentiation and grain yield formation of summer maize in the field. International Journal of Biometeorology 59, 1189-1200.

Doebley J, Stec A, Hubbard L. 1997. The evolution of apical dominance in maize. Nature 386, 485488.

Franklin KA. 2008. Shade avoidance. New Phytologist 179, 930-944.

Gangappa SN, Botto JF. 2014. The BBX family of plant transcription factors. Trends in Plant Science 19, 460-470.

Gonzalez-Grandio E, Pajoro A, Franco-Zorrilla JM, Tarancon C, Immink RG, Cubas P. 2017. Abscisic acid signaling is controlled by a BRANCHED1/HD-ZIP I cascade in Arabidopsis axillary buds. Proceedings of the National Academy of Sciences of the United States of America 114, E245-e254.

Hornitschek P, Kohnen MV, Lorrain S, et al. 2012. Phytochrome interacting factors 4 and 5 control seedling growth in changing light conditions by directly controlling auxin signaling. The Plant Journal 71, 699-711.

Hornitschek P, Lorrain S, Zoete V, Michielin O, Fankhauser C. 2009. Inhibition of the shade avoidance response by formation of non - DNA binding bHLH heterodimers. The Embo Journal 28, 3893.

Jin M, Liu X, Jia W, et al. 2018. ZmCOL3, a CCT gene represses flowering in maize by interfering with the circadian clock and activating expression of $\mathrm{ZmCCT}$. Journal of Integrative Plant Biology 60, $465-480$

Kebrom TH, Mullet JE. 2016. Transcriptome profiling of tiller buds provides new insights into PhyB regulation of tillering and indeterminate growth in Sorghum. Plant Physiology 170, 2232-2250.

Keuskamp DH, Sasidharan R, Pierik R. 2010. Physiological regulation and functional significance of shade avoidance responses to neighbors. Plant Signaling \& Behavior 5, 655-662.

Kircher S, Kozma-Bognar L, Kim L, Adam E, Harter K, Schafer E, Nagy F. 1999. Light qualitydependent nuclear import of the plant photoreceptors phytochrome A and B. The Plant Cell 11, 
1445-1456.

Kong F, Zhang T, Liu J, et al. (2017) Regulation of leaf angle by auricle development in Maize. Molecular Plant 10, 516-519.

Kumar I, Swaminathan K, Hudson K, Hudson ME. 2016. Evolutionary divergence of phytochrome protein function in Zea mays PIF3 signaling. Journal of Experimental Botany 67, 4231-4240.

Kurepin LV, Emery RJ, Pharis RP, Reid DM. 2007. Uncoupling light quality from light irradiance effects in Helianthus annuus shoots: putative roles for plant hormones in leaf and internode growth. Journal of Experimental Botany 58, 2145-2157.

Lee N, Choi G. 2017. Phytochrome-interacting factor from Arabidopsis to liverwort. Current Opinion in Plant Biology 35, 54-60.

Leivar P, Monte E, Oka Y, Liu T, Carle C, Castillon A, Huq E, Quail PH. 2008. Multiple phytochrome-interacting bHLH transcription factors repress premature seedling photomorphogenesis in darkness. Current Biology 18, 1815-1823.

Leivar P, Quail PH. 2011. PIFs: pivotal components in a cellular signaling hub. Trends in Plant Science 16, 19-28.

Li J, Li G, Wang H, Wang Deng, X. 2011. Phytochrome signaling mechanisms. Arabidopsis Book 9 , $\mathrm{e} 0148$.

Li L, Ljung K, Breton G, et al. 2012. Linking photoreceptor excitation to changes in plant architecture. Genes \& Development 26, 785-790.

Liu H, Yang C, Li L. 2016. Shade-induced stem elongation in rice seedlings: implication of tissuespecific phytohormone regulation. Journal of Integrative Plant Biology 58, 614-617.

Lorrain S, Allen T, Duek PD, Whitelam GC, Fankhauser C. 2008. Phytochrome-mediated inhibition of shade avoidance involves degradation of growth-promoting bHLH transcription factors. The Plant Journal 53, 312-323.

Ma L, Xue N, Fu X, Zhang H, Li G. 2017. Arabidopsis thaliana FAR-RED ELONGATED HYPOCOTYLS3 (FHY3) and FAR-RED-IMPAIRED RESPONSE1 (FAR1) modulate starch synthesis in response to light and sugar. New Phytologist 213, 1682-1696.

Maddonni G, Otegui ME, Cirilo AG. 2001. Plant population density, row spacing and hybrid effects on maize canopy architecture and light attenuation. Field Crops Research 71, 183-193.

Page E, Tollenaar M, Lee E, Lukens L, Swanton C. 2010. Shade avoidance: an integral component of crop-weed competition. Weed Research 50, 281-288.

Pfeiffer A, Shi H, Tepperman JM, Zhang Y, Quail PH. 2014. Combinatorial complexity in a transcriptionally centered signaling hub in Arabidopsis. Molecular Plant 7, 1598-1618.

Phillips KA, Skirpan AL, Liu X, Christensen A, Slewinski TL, Hudson C, Barazesh S, Cohen JD, Malcomber S, McSteen P. 2011. Vanishing tassel 2 encodes a grass-specific tryptophan aminotransferase required for vegetative and reproductive development in maize. The Plant Cell 23, 550-566.

Pignon CP, Jaiswal D, McGrath JM, Long SP. 2017. Loss of photosynthetic efficiency in the shade. An Achilles heel for the dense modern stands of our most productive C4 crops? Journal of Experimental Botany 68, 335-345.

Reed JW, Nagpal P, Poole DS, Furuya M, Chory J. 1993. Mutations in the gene for the red/far-red light receptor phytochrome $\mathrm{B}$ alter cell elongation and physiological responses throughout Arabidopsis development. The Plant Cell 5, 147-157.

Robson P, Whitelam GC, Smith H. 1993. Selected components of the shade-avoidance syndrome are 
displayed in a normal manner in mutants of Arabidopsis thaliana and Brassica rapa deficient in phytochrome B. Plant Physiology 102, 1179-1184.

Roigvillanova I, Bou J, Sorin C, Devlin PF, Martínezgarcía JF. 2006. Identification of primary target genes of phytochrome signaling. Early transcriptional control during shade avoidance responses in Arabidopsis. Plant Physiology 141, 85-96.

Sharwood RE, Sonawane BV, Ghannoum O. 2014. Photosynthetic flexibility in maize exposed to salinity and shade. Journal of Experimental Botany 65, 3715-3724.

Sheehan MJ, Kennedy LM, Costich DE, Brutnell TP. 2007. Subfunctionalization of PhyB1 and PhyB2 in the control of seedling and mature plant traits in maize. The Plant Journal 49, 338353.

Shi Q, Zhang H, Song X, Jiang Y, Liang R, Li G. 2018. Functional characterization of the maize phytochrome-interacting factors PIF4 and PIF5. Frontiers in Plant Science 8, 2273.

Sorin C, Salla-Martret M, Bou-Torrent J, Roig-Villanova I, Martinez-Garcia JF. 2009. ATHB4, a regulator of shade avoidance, modulates hormone response in Arabidopsis seedlings. The Plant Journal 59, 266-277.

Takeda T, Suwa Y, Suzuki M, Kitano H, Ueguchi-Tanaka M, Ashikari M, Matsuoka M, Ueguchi C. 2003. The OSTB1 gene negatively regulates lateral branching in rice. The Plant Journal 33, 513-520.

Wang H, Wu G, Zhao B, Wang B, Lang Z, Zhang C, Wang H. 2016. Regulatory modules controlling early shade avoidance response in maize seedlings. BMC Genomics 17, 269.

Wang H, Zhang Z, Li H, Zhao X, Liu X, Ortiz M, Lin C, Liu B. 2013. CONSTANS-LIKE 7 regulates branching and shade avoidance response in Arabidopsis. Journal of Experimental Botany 64, 1017-1024.

Whipple CJ, Kebrom TH, Weber AL, Yang F, Hall D, Meeley R, Schmidt R, Doebley J, Brutnell TP, Jackson DP. 2011. Grassy tillers1 promotes apical dominance in maize and responds to shade signals in the grasses. Proceedings of the National Academy of Sciences of the United States of America 108, E506-512.

Zhang Y, Mayba O, Pfeiffer A, Shi H, Tepperman JM, Speed TP, Quail PH. 2013. A quartet of PIF bHLH factors provides a transcriptionally centered signaling hub that regulates seedling morphogenesis through differential expression-patterning of shared target genes in Arabidopsis. PLoS Genetics 9, e1003244.

Zhu J, Vos J, van der Werf W, van der Putten PEL, Evers JB. 2014. Early competition shapes maize whole-plant development in mixed stands. Journal of Experimental Botany 65, 641-653. 
bioRxiv preprint doi: https://doi.org/10.1101/397596; this version posted August 22, 2018. The copyright holder for this preprint (which was not certified by peer review) is the author/funder. All rights reserved. No reuse allowed without permission.

Table 1. Core responsible genes involved in regulating the SAS in maize.

\begin{tabular}{|c|c|c|c|c|c|c|c|c|c|c|c|}
\hline Gene ID & Gene name & Fo & F1 & $\mathbf{F 3}$ & $\mathbf{R 1}$ & Function & Homologous & sha_0h & sha_1h & sha_3h & sha_6h \\
\hline GRMZM2G157727 & PHYAI & 67.86 & 66.74 & 92.78 & 98.20 & \multirow{9}{*}{ light signal } & AT1G09570 & 11.27 & 13.05 & 22.31 & 26.31 \\
\hline GRMZM2G181028 & PHYA2 & 18.04 & 17.11 & 28.16 & 35.46 & & AT1G09570 & 4.64 & 6.54 & 16.06 & 21.57 \\
\hline GRMZM2G124532 & PHYBI & 11.40 & 17.86 & 35.34 & 34.59 & & AT2G18790 & 7.42 & 10.24 & 14.09 & 15.58 \\
\hline GRMZM2G057935 & PHYCl & 7.59 & 13.02 & 16.97 & 18.75 & & AT5G35840 & 13.52 & 16.15 & 21.70 & 26.56 \\
\hline GRMZM2G137046 & HY5 & 27.90 & 16.12 & 9.37 & 12.25 & & AT5G11260 & 14.86 & 10.84 & 15.36 & 12.65 \\
\hline GRMZM2G016756 & PIFl & 1.87 & 1.38 & 5.01 & 12.58 & & AT2G20180 & 7.88 & 3.09 & 5.92 & 5.49 \\
\hline GRMZM2G107945 & $F K F 1$ & 2.46 & 6.73 & 22.40 & 34.81 & & AT1G68050 & 0.41 & 1.09 & 7.21 & 42.68 \\
\hline GRMZM2G172506 & NPY5 & 10.49 & 24.22 & 27.95 & 31.63 & & AT4G37590 & 8.60 & 10.87 & 19.53 & 29.57 \\
\hline GRMZM2G176506 & $P L P B$ & 7.55 & 11.82 & 20.13 & 17.42 & & AT2G02710 & 2.05 & 4.13 & 7.28 & 10.46 \\
\hline GRMZM2G127308 & $V T 2 / T A A 1$ & 8.05 & 15.64 & 32.64 & 10.47 & \multirow{3}{*}{ auxin } & AT4G24670 & & & & \\
\hline GRMZM2G160005 & IAA22 & 1.91 & 4.88 & 10.12 & 10.28 & & AT1G19220 & 10.11 & 14.12 & 18.63 & 23.67 \\
\hline GRMZM2G159285 & IAA16 & 22.51 & 24.54 & 52.64 & 57.21 & & AT3G04730 & 74.31 & 87.72 & 164.6 & 258.3 \\
\hline GRMZM2G382569 & SRGI & 29.84 & 35.33 & 84.30 & 67.89 & \multirow{3}{*}{ ethylene } & AT1G17020 & 3.51 & 3.50 & 6.58 & 12.32 \\
\hline GRMZM2G055180 & ERF9 & 12.33 & 33.65 & 21.19 & 22.60 & & AT5G47220 & 11.69 & 17.49 & 14.88 & 17.51 \\
\hline GRMZM2G111415 & ERF10 & 7.37 & 18.71 & 22.28 & 11.27 & & AT5G25190 & 5.69 & 5.44 & 4.69 & 5.08 \\
\hline GRMZM2G177104 & GA2ox8 & 0.55 & 1.94 & 4.33 & 3.23 & \multirow{2}{*}{ GA } & AT4G21200 & 0.02 & 0.04 & 0.22 & 0.08 \\
\hline GRMZM2G368411 & GA20oxl & 2.78 & 5.13 & 12.54 & 3.07 & & AT4G25420 & 3.29 & 11.48 & 20.89 & 28.73 \\
\hline GRMZM2G005624 & $G T 1$ & 7.24 & 3.65 & 3.31 & 2.09 & \multirow{18}{*}{$\begin{array}{l}\text { transcript- } \\
\text { ion factors }\end{array}$} & AT4G36740 & & & & \\
\hline AC233950.1 & $T B 1$ & 1.09 & 0.22 & 0 & 0.16 & & AT3G18550 & & & & \\
\hline GRMZM2G044752 & $H B 53$ & 0.45 & 2.74 & 2.72 & 0.96 & & AT2G44910 & 3.09 & 10.16 & 9.19 & 7.06 \\
\hline GRMZM2G159996 & $B B X 13$ & 7.75 & 14.81 & 27.37 & 33.38 & & AT1G28050 & 3.95 & 5.24 & 7.57 & 13.88 \\
\hline GRMZM2G110541 & $B B X 22$ & 0.59 & 1.74 & 7.37 & 3.80 & & AT4G39070 & 2.43 & 5.90 & 4.99 & 4.64 \\
\hline GRMZM2G018876 & $B B X 24$ & 38.82 & 49.08 & 139.4 & 102.7 & & AT1G06040 & 128.5 & 161.5 & 277.1 & 340.5 \\
\hline GRMZM2G057955 & MYB3 & 13.52 & 27.32 & 160.0 & 36.41 & & AT4G01060 & 31.06 & 78.03 & 112.3 & 118.7 \\
\hline GRMZM2G114503 & $R L 6$ & 166.3 & 103.0 & 36.15 & 27.05 & & AT1G75250 & 39.91 & 25.22 & 14.75 & 12.70 \\
\hline GRMZM2G145041 & RVEl & 28.41 & 11.63 & 13.97 & 13.22 & & AT5G17300 & 21.40 & 13.76 & 10.30 & 3.32 \\
\hline GRMZM2G150260 & $R L 1$ & 18.58 & 6.52 & 0.99 & 2.04 & & AT4G39250 & 16.41 & 8.73 & 3.67 & 2.75 \\
\hline GRMZM2G042895 & bHLH116 & 5.73 & 40.08 & 10.36 & 8.07 & & AT4G29930 & 0.18 & 1.19 & 0.43 & 0.30 \\
\hline GRMZM2G445634 & $J A Z 1$ & 9.56 & 33.31 & 12.63 & 8.91 & & AT1G19180 & 5.56 & 6.64 & 4.55 & 5.29 \\
\hline GRMZM2G138455 & $C D F 2$ & 21.34 & 12.39 & 9.72 & 14.31 & & AT5G39660 & 10.15 & 7.77 & 5.75 & 2.67 \\
\hline GRMZM2G148453 & TOCl & 0.89 & 2.63 & 8.60 & 12.53 & & AT5G61380 & 0.58 & 0.33 & 1.73 & 4.63 \\
\hline GRMZM2G367834 & PRR5 & 4.39 & 5.26 & 12.09 & 16.41 & & AT5G24470 & 1.61 & 2.20 & 8.96 & 16.48 \\
\hline GRMZM2G081949 & REM4 & 52.17 & 169.8 & 183.7 & 187.3 & & AT2G41870 & 41.31 & 58.88 & 52.43 & 60.92 \\
\hline GRMZM2G086876 & $A H L 9$ & 6.77 & 12.18 & 13.84 & 12.06 & & AT2G45850 & 13.85 & 14.80 & 18.59 & 29.35 \\
\hline GRMZM2G071042 & SAP5 & 44.10 & 88.40 & 143.2 & 76.58 & & AT3G12630 & 41.14 & 47.85 & 52.63 & 48.19 \\
\hline GRMZM2G094990 & EXPBI & 120.0 & 93.09 & 103.4 & 50.16 & cell wall & AT1G65680 & 129.5 & 113.3 & 67.47 & 38.84 \\
\hline GRMZM2G005840 & XERICO & 82.40 & 128.9 & 130.3 & 67.80 & \multirow{2}{*}{$\begin{array}{c}\text { protein } \\
\text { degradation }\end{array}$} & AT2G04240 & 15.86 & 39.81 & 55.30 & 71.83 \\
\hline GRMZM2G390436 & DAFLI & 1.68 & 4.97 & 10.86 & 8.04 & & AT3G10910 & 1.88 & 2.27 & 4.39 & 8.14 \\
\hline
\end{tabular}

536 Note: Sha_0h, sha_1h, sha_3h and sha_6h are the RPKM of the genes in maize treated by shade for $0 \mathrm{~h}, 1 \mathrm{~h}, 3 \mathrm{~h}$ and $6 \mathrm{~h}$, respectively, in previous study (Wang 
(a)

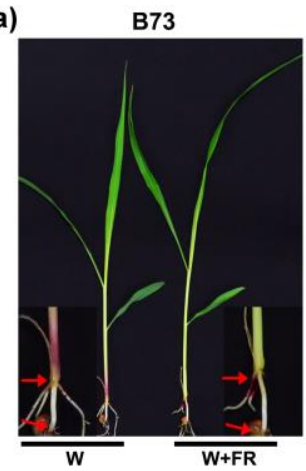

(b)
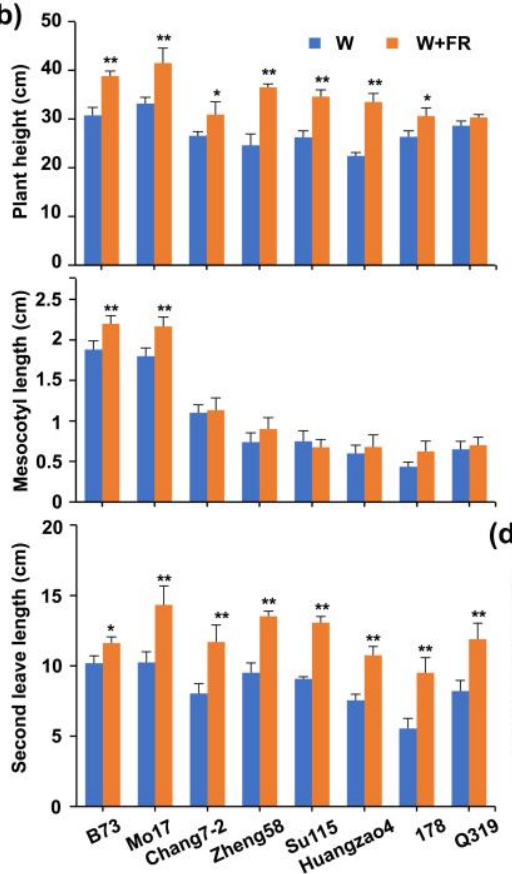

Mo17

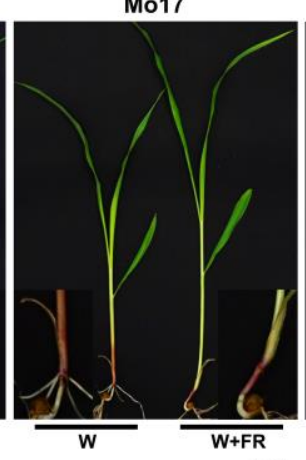

(c)
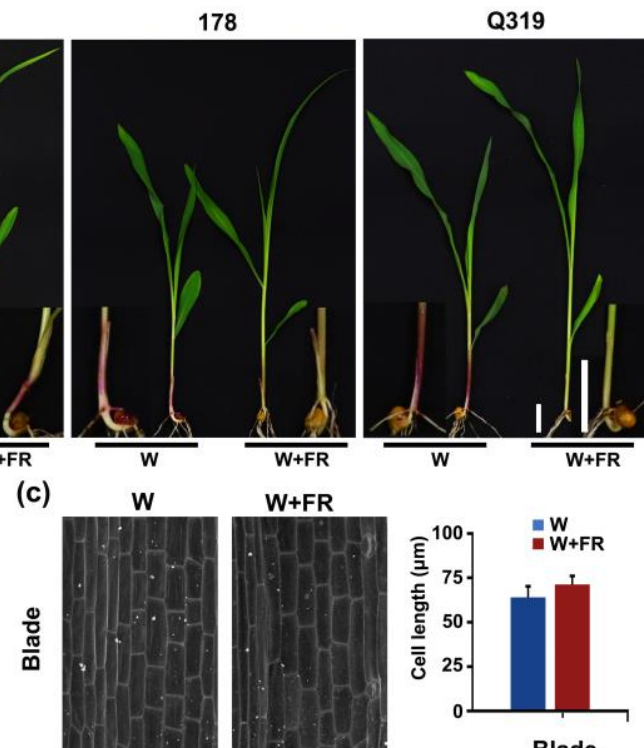

W+FR
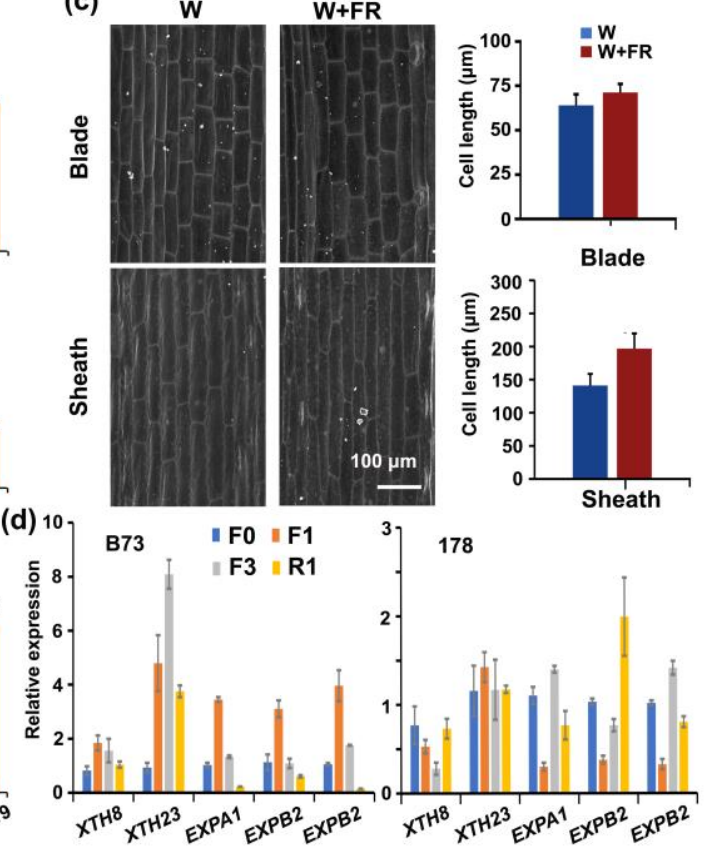

Fig. 1. The phenotype of maize seedlings grown under high or low R:FR conditions.

542 (a) The phenotypes of B73, Mo17, 178, and Q319 seedlings grown under high R:FR

543 (13.3) and low R: FR light (0.19). Scale bar, $3 \mathrm{~cm}$.

544 (b) The plant height, mesocotyl length, and second-leaf length of different inbred lines.

545 Data represent the mean and $\mathrm{SD}$ of at least 30 seedlings. $* \mathrm{P}<0.05, * * \mathrm{P}<0.01$

(c) SEM and cell length analysis of the blade and sheath tissues of inbred B73 grown under high or low R:FR conditions. Data represent the mean and SD of at least 100 cells. $* \mathrm{P}<0.05, * * \mathrm{P}<0.01$

549 (d) RT-qPCR analysis of the transcription level of cell elongation-related genes in B73 and 178 treated with far red light for $0 \mathrm{~h}(\mathrm{~F} 0), 1 \mathrm{~h}(\mathrm{~F} 1)$, and $3 \mathrm{~h}(\mathrm{~F} 3)$, and then with red

551 light for $1 \mathrm{~h}(\mathrm{R} 1)$, respectively. Data are means and SD of three independent biological replicates. 
(a)

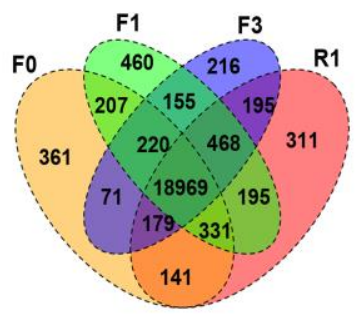

(b)

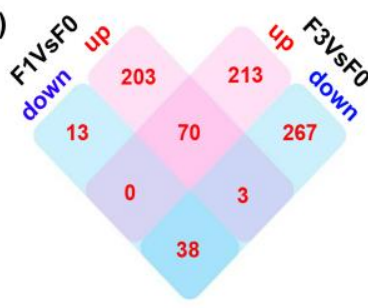

(c)

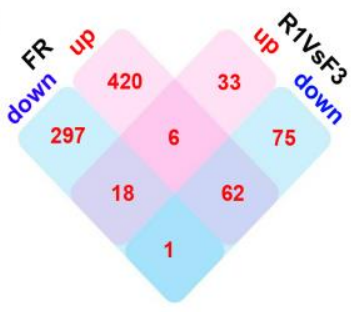

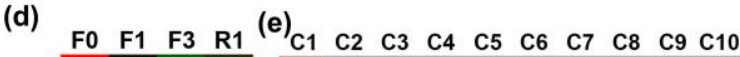

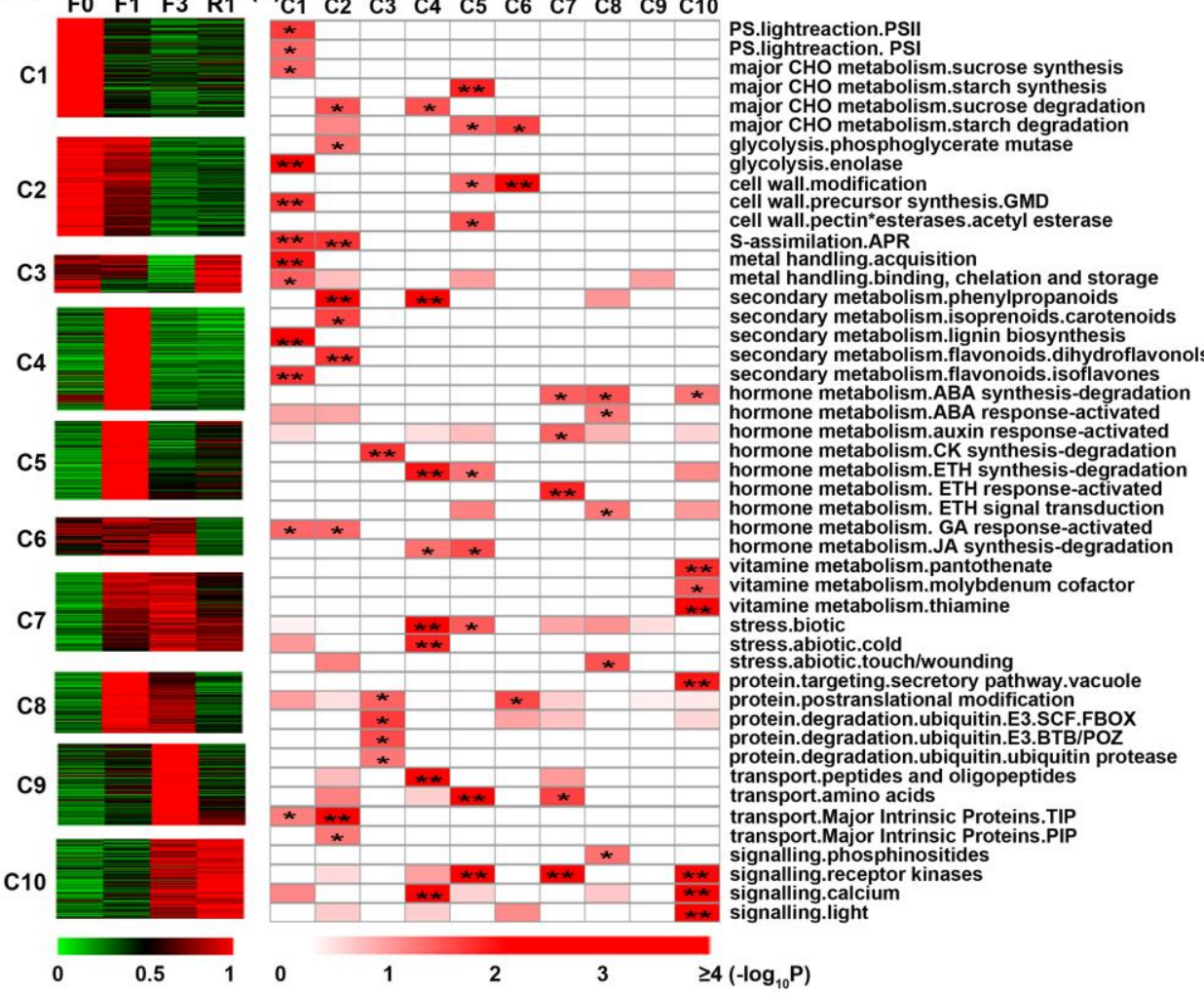

Fig. 2. Transcriptome analysis of maize seedling responses to simulated shade.

(a) Venn diagram of the numbers of the expressed genes in B73 seedlings treated with

556 far red light for $0 \mathrm{~h}(\mathrm{~F} 0), 1 \mathrm{~h}(\mathrm{~F} 1)$, and $3 \mathrm{~h}(\mathrm{~F} 3)$, and then with red light for $1 \mathrm{~h}(\mathrm{R} 1)$.

557 (b) Venn diagram of the numbers of DEGs between F1 and F0 (F1 vs. F0) and F3 and

558 F0 (F3 vs. F0), respectively.

(c) Venn diagram of the numbers of the FR DEGs and DEGs between R1 and F3 (R1

vs. F3). FR DEGs refer to the DEGs of F1 vs. F0 and F3 vs. F0, excluding the 3 genes

561 showing different trends.

562 (d) Ten expression clusters of DEGs (C1-C10), ordered according to the time points

563 of their peak expression. For each gene, the normalized values are shown.

564 (e) Mapman functional enrichment analysis of DEGs. Fisher's exact test was used to

565 determine whether a functional category was enriched. *, $q<0.05 ;{ }^{* *} q<0.01$ 
(a) F0 F1 F3 R1

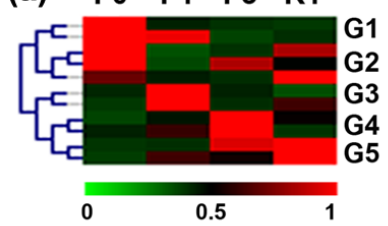

(b)

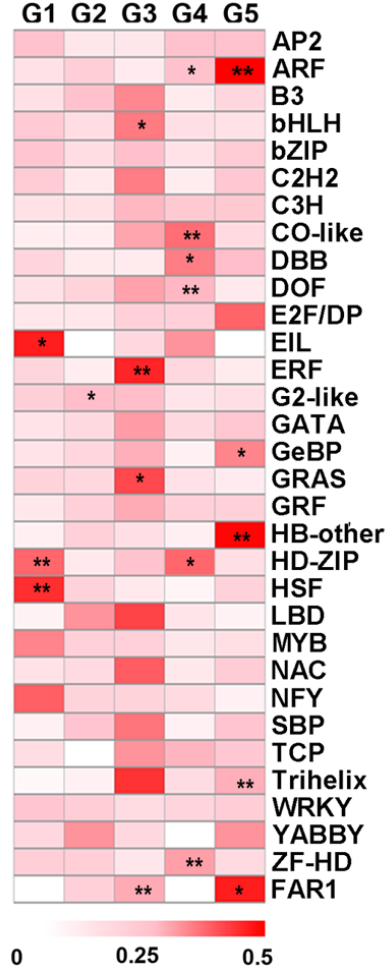

567 Fig. 3. Transcription factor family enrichment analysis.

568 (a) Five expression groups (G1-G5) of the expressed transcription factors.

569 (b) Transcription factor family enrichment analysis. The values shown are the number

570 of transcription factor family members classified in a cluster: the total number of

571 transcription factor family members. Fisher's exact test was used to determine whether

572 a transcription factor family was enriched. *, $q<0.05 ;{ }^{* *} q<0.01$. 
(a)

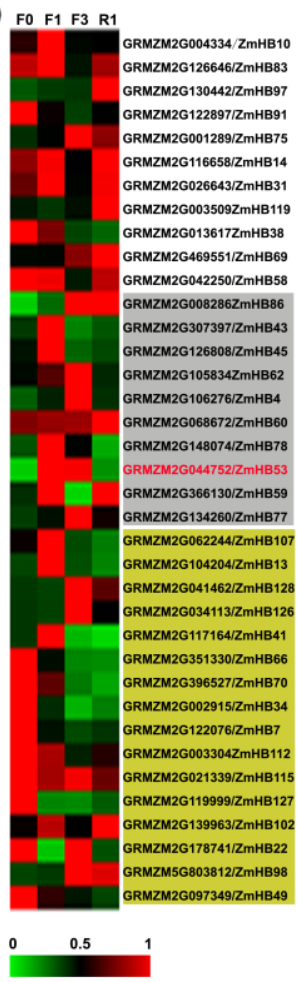

(b)

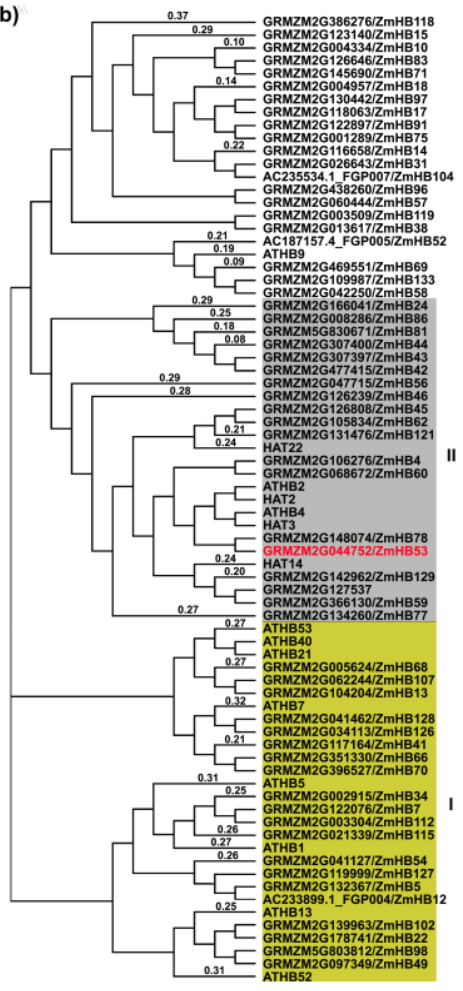

(c)

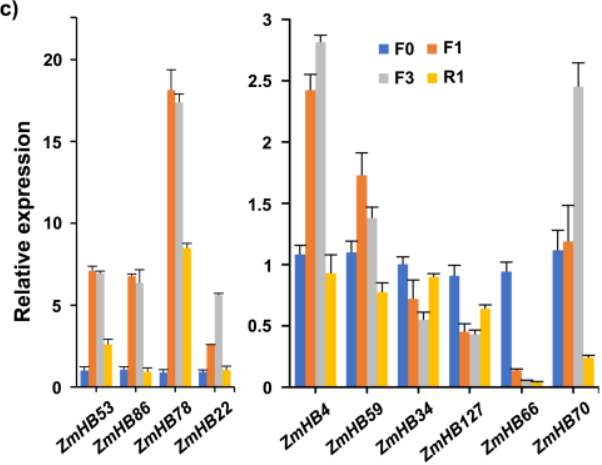

(d).

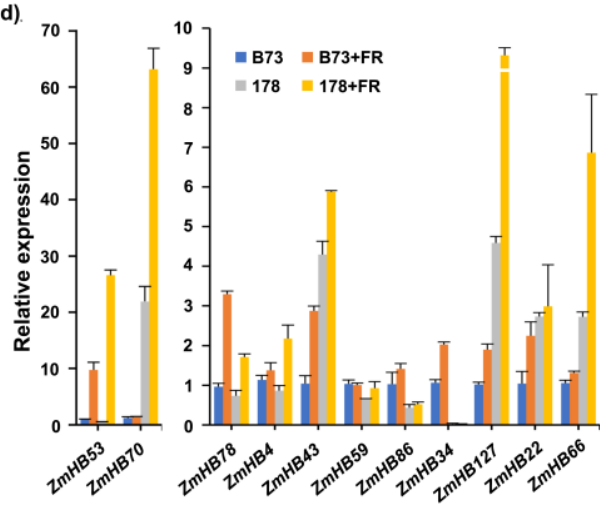

Fig. 4. Expression pattern analysis of HD-ZIP members in shade response

(a) Heat map representation of the expression patterns of HD-ZIPs. For each gene, the value shown is the RPKM value normalized by the maximum values of all RPKM values of the gene in F0, F1, F3 and R1. The gene in gray background belong to type II HD-ZIPS, while in yellow background belong to type I HD-ZIPS.

(b) Phylogenetic tree of selected HD-ZIP family proteins in Zea mays (Zm) and Arabidopsis thaliana (At). The neighbor-joining method was used to construct the phylogenetic tree. (c) RT-qPCR analyses revealed that selected HD-ZIP family genes were rapidly induced or reduced by far-red or red light. Three-leaf stage seedling plants of maize inbred line B73 were used to harvest second leaves, and then used to perform RT-qPCR analysis. Actin was used as an internal control for RT-qPCR analysis. Data are means and SD of three independent biological replicates.

(d) RT-qPCR analyses of the expression of selected HD-ZIP family genes in B73 and 178 lines treated by white or FR for $1 \mathrm{~h}$. 
(a)

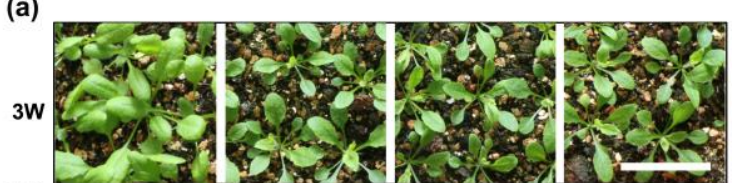

(b)

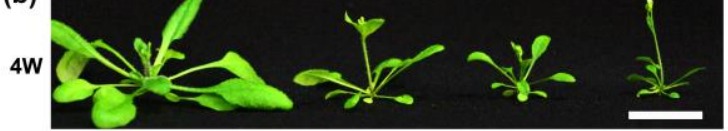

(c)

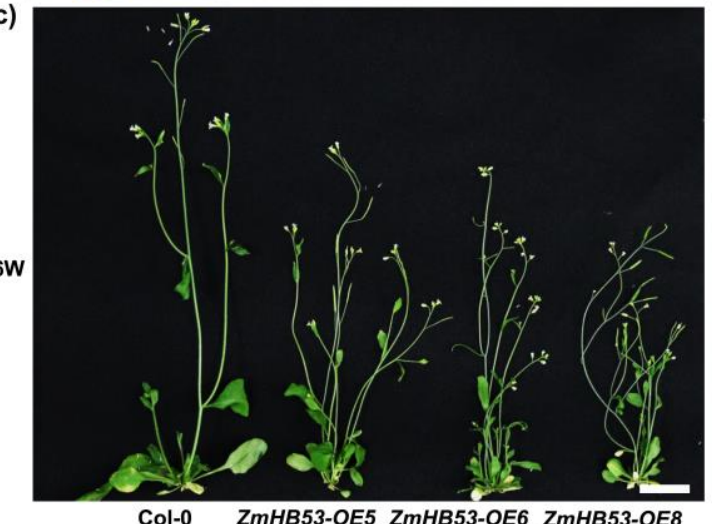

(d)
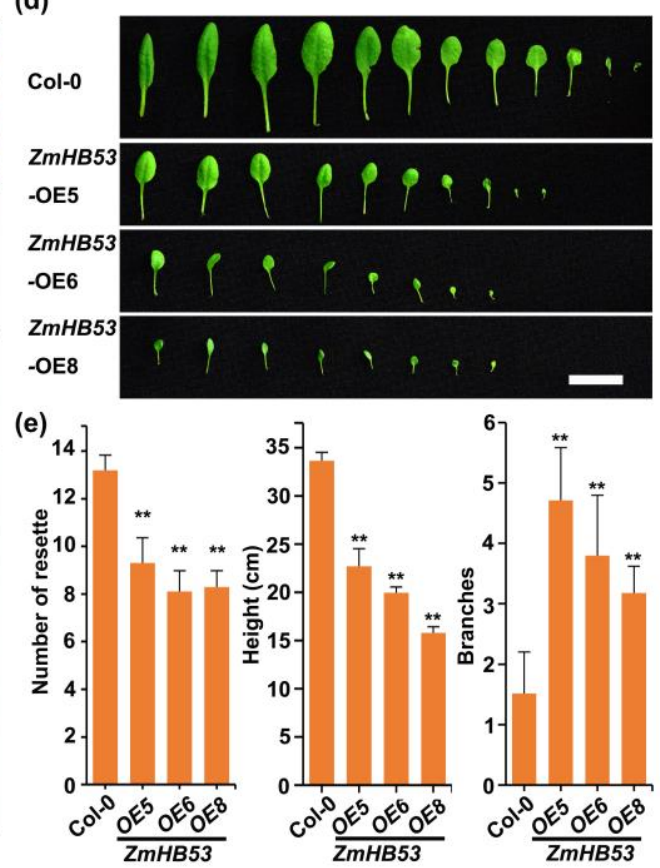

(f)

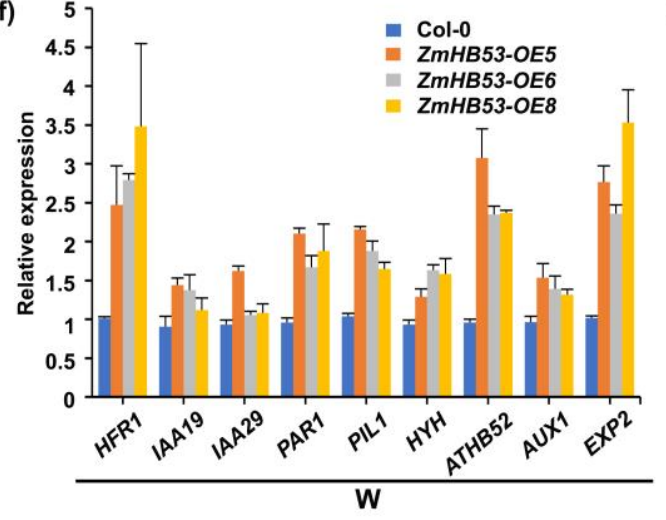

(g) 25

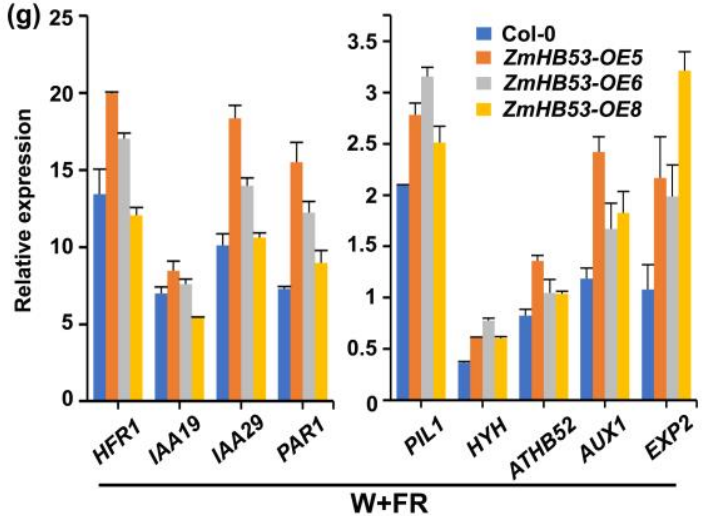

Fig. 5. Overexpression of ZmHB53 in wild-type Arabidopsis Col-0 plants.

(a-c), Phenotype of 3-, 4-, and 6-week-old Arabidopsis overexpressing ZmHB53, respectively. Scale bar, $2 \mathrm{~cm}$.

(d) Leaves of 4-week-old Arabidopsis overexpressing ZmHB53. Scale bar, $2 \mathrm{~cm}$.

(e) Quantification of rosette leaf number shown in B and D, plant height and number of branches shown in C. Scale bar, $2 \mathrm{~cm}$. $* P<0.05 ; * P<0.01 ; n=20$.

(f-g) RT-qPCR analysis of the expression of selected shade-response genes in Arabidopsis overexpressing ZmHB53. Seven-day-old seedlings grown under LD (W, high R:FR; W+FR, low R:FR) conditions were used. UBQ1 was used as the internal control. Data are means and SD of three replicates. 